\title{
Relative contributions of neighbourhood and animal movements to Coxiella burnetii infection in dairy cattle herds
}

\author{
Simon Nusinovici ${ }^{1,2}$, Thierry Hoch $^{1,2}$, Stefan Widgren ${ }^{3}$, Alain Joly ${ }^{1,2,4}$, Ann Lindberg 3 , François \\ Beaudeau ${ }^{1,2}$ \\ ${ }^{1}$ INRA, UMR1300 Biology, Epidemiology and Risk Analysis in Animal Health, Nantes, France; ${ }^{2}$ LUNAM \\ Université, Oniris, UMR BioEpAR, Nantes, France; ${ }^{3}$ National Veterinary Institute, Sweden; ${ }^{4} U B G D S$ (Union \\ Bretonne des Groupement de Défense Sanitaire), Vannes, France
}

\begin{abstract}
Q fever in dairy cattle herds occurs mainly after inhalation of contaminated aerosols generated from excreta by shedder animals. Propagation of Coxiella burnetii, the cause of the disease between ruminant herds could result from transmission between neighbouring herds and/or the introduction of infected shedder animals in healthy herds. The objective of this study were (i) to describe the spatial distribution C. burnetii-infected dairy cattle herds in two different regions: the Finistère District in France (2,829 herds) and the island of Gotland in Sweden (119 herds) and (ii) to quantify and compare the relative contributions of C. burnetii transmission related to neighbourhood and to animal movements on the risk for a herd to be infected. An enzyme - linked immunosorbent assay was used for testing bulk tank milk in May 2012 and June 2011, respectively. Only one geographical cluster of positive herds was identified in north-western Finistère. Logistic regression was used to assess the association of risk for a herd to test positively with local cattle density (the total number of cattle located in a $5 \mathrm{~km}$ radius circle) and the in-degree (ID) parameter, a measure of the number of herds from which each herd had received animals directly within the last 2 years. The risk for a herd to test positively was higher for herds with a higher local cattle density [odds ratio $(\mathrm{OR})=2.3,95 \%$ confidence interval $(\mathrm{CI})=1.6-3.2$, for herds with a local density between 100 and 120 compared to herds with a local density 60]. The risk was also higher for herds with higher IDs (OR = 2.3, $95 \% \mathrm{CI}=1.6-3.2$, for herds with ID 3 compared to herds that did not introduce animals). The proportion of cases attributable to infections in the neighbourhood in high-density areas was twice the proportion attributable to animal movements, suggesting that wind plays a main role in the transmission.
\end{abstract}

Keywords: Q fever, epidemiology, risk factor, spatial clustering, control measures, France, Sweden.

\section{Introduction}

Numerous species, including humans, can be infected by Coxiella burnetii, an obligate, intracellular bacterial pathogen responsible for Q fever, a worldwide zoonosis. Infection in humans is usually asymptomatic but can induce acute or chronic disease (ArricauBouvery and Rodolakis, 2005). In livestock, which is considered the main reservoir, C. burnetii infection can lead to abortion, stillbirth and metritis and is shed through birth products, faeces, urine, milk and vaginal mucus (Berri et al., 2000; Guatteo et al., 2006, 2007). The infection occurs mainly after inhalation of aerosols contaminated by shedder ruminants. C. burnetii is highly resistant in the environment, which thus

\footnotetext{
Corresponding author:

Simon Nusinovici

INRA Oniris, UMR1300 Biology

Epidemiology and Risk Analysis in Animal Health (BioEpAR)

La Chantrerie, CS 40706, 44307 Nantes, France

Tel. +332 4068-4028; Fax +332 4068-7768

E-mail: simon.nusinovici@oniris-nantes.fr
}

represents an important source of contamination (Welsh et al., 1959).

C. burnetii propagation between ruminant herds could result from transmission between neighbouring herds, e.g. through wind, and/or the introduction of infected shedder animals in healthy herds. Wind has been identified as playing a significant role in the transmission between sheep and humans (TissotDupont et al., 1999, 2004), while movement of infected animals clearly contributes to the bacterial spread between herds. Although both factors probably influence the transmission of the bacteria, their relative impacts have not been investigated as far as we know.

The spatial distribution of $\mathrm{Q}$ fever infection probably depends on the relative contribution of different transmission routes. Our hypothesis is that transmission from infected neighbourhoods (e.g. by wind) does not lead to distributions resembling those correlated with what happens after animal exchange between herds. The presence of clusters of infected herds would indirectly suggest the main reason for local spread. The extent at which infected herds 
would be evenly distributed depends on the contact network of animal movements. If wind had a powerful effect in spreading the disease, a high density of herds should strongly influence local transmission of the bacterium between herds. To investigate this assumption, two areas were selected: one in France (the Finistère District) and one in Sweden (the Island of Gotland). Both areas are highly infected but differ with respect to cattle density.

The objectives of this study were (i) to describe the spatial distribution of $\mathrm{Q}$ fever-infected dairy herds in two different regions and (ii) to quantify and compare the relative contributions of C. burnetii transmission related to neighbourhood and to animal movements on the risk for a herd to be infected.

\section{Materials and methods}

\section{Study area and available data}

Two areas were considered: the Finistère District in western France (the French territory is divided into 95 districts) and the island of Gotland in Sweden. In Finistère, bulk tank milk (BTM) was systematically collected in May 2012 in all dairy herds $(\mathrm{n}=2,829)$ and tested using the LSI Q fever enzyme - linked immunosorbent assay (ELISA) $\mathrm{kit}^{\circledR}$ (LSI; Lissieu, France) for detection of antibodies against C. burnetii (cut-off for positivity at $>40$ titre).

On Gotland, 119 out of 246 dairy producers accepted to have their herd tested for C. burnetii antibody in June 2011 using the IDEXX Chekit ${ }^{\circledR}$ Q fever kit (IDEXX Laboratories; Westbrook, USA) (cut-off for positivity at $>40$ titre).

All commercial animal movements were available from 2000 to 2011 in France and from 2005 to 2011 in Sweden. In both countries, the geographical coordinates of all the cattle herds were available for georeferencing. To calculate the local cattle density, the number of animals present in all herds (not only the tested ones) in the year before the ELISA test was considered.

The status of each herd with regard to C. burnetii infection was assessed by antibody detection in BTM.

\section{Local cattle density}

The local cattle density was used as a proxy to measure the proximity between cattle herds. It was calculated for a $5 \mathrm{~km}$ radius circle around each herd. Both beef and dairy herds were considered because they could both contribute to the spread of C. burnetii $(62 \%$ and $33 \%$ of the cattle herds were dairy herds in Finistère in France and on the island of Gotland in Sweden, respectively). The density was calculated at the animal level because the herd size could influence the risk of infection. For instance, a herd could have a higher risk of infection if located close to a large infected herd compared to a small one. For herds located less than $5 \mathrm{~km}$ from the border (administrative border or sea), the local cattle density was approximated using the local cattle density of the nearest herd located more than $5 \mathrm{~km}$ from the border. Forty-five and $48 \%$ of the cattle herds were located less than $5 \mathrm{~km}$ from the borders in the Finistère and on the island of Gotland, respectively. The median distance between herds close to the borders and herds used for the approximation was less than $4 \mathrm{~km}$ in both study areas.

\section{Animal movements}

Network analysis was used to investigate the impact of animal movements on the risk for a herd to be detected as ELISA positive. Among network parameters, the in-degree (ID) was used, a measure of the number of herds from which each herd receives animals (Wasserman et al., 1994); in our case, the number of herds from which each herd received animals directly. This network parameter has been used in several studies to investigate animal movements in the context of disease control and risk-based surveillance (Christley et al., 2005; Nöremark et al., 2011; Frössling et al., 2012). A time window has to be defined for the calculation of the ID. Here, the window corresponded to the period prior to the ELISA testing during which animal movements could have played a role in the transmission of the bacterium between herds. A statistical approach was used to select this time window, the same for each area (France and Sweden). It consisted in the following steps:

(i) calculation of IDs during different periods: 1, 2, 3 and 4 years before the date of the ELISA testing;

(ii) construction of four logistic regression models assessing the relation between the risk for a herd to be detected as positive and each of the four IDs variables; and

(iii) calculation of the associated odds ratio (OR) and the Akaike information criterion (AIC) for each model.

The selection of the periods for the calculation of 
the IDs was based on the OR and the AIC values, but because the models were not nested, it was not possible to calculate the p-values associated with each AIC. However, a large decrease in AIC between two models still corresponds to an increase in the goodness of fit. The principle was to select the model with the minimum AIC and the maximum OR. On the one hand, the model with the minimum AIC corresponds to the highest likelihood model, while on the other, the model with the maximum OR corresponds to the highest explanatory model, i.e. the model which best describes the effect of animal movements with regard to the risk of becoming infected.

\section{Spatial analysis}

The spatial analysis consisted in the detection of putative clusters of BTM-positive herds by spatial statistical analysis using a Bernoulli model in SaTScan (Kulldorff, 1997; Kulldorff and Information Management Services, 2009). Analyses were performed separately for the French and the Swedish areas.

\section{Risk factor analysis}

The risk for a herd to be detected as a carrier of antibodies against C. burnetii in BTM in relation to the two independent variables was assessed using logistic regression:

$$
\begin{gathered}
\operatorname{logit} p(x)=\log \left(\frac{p(x)}{1-p(x)}\right) \\
=\alpha+\beta_{1} D E N S+\beta_{2} I D+\beta_{3} C O
\end{gathered}
$$

(equation 1)

where $p(x)$ is the risk for a herd to be detected as positive, $\alpha$ the intercept, DENS the local cattle density variable ( 5 classes), ID the in-degree parameter variable ( 3 classes) and $\mathrm{CO}$ the country variable $(2$ classes). $\beta_{1}, \beta_{2}$ and $\beta_{3}$ are the adjusted regression coefficients associated with the variables estimated by the model. Logistic regressions were performed using $\mathrm{R}$ software ( $\mathrm{R}$ Development Core Team, 2012). ID parameters were calculated using the "EpiContactTrace" package ${ }^{1}$ of R software.

${ }^{1}$ Stefan Widgren and Maria Noremark, 2012. EpiContactTrace: Epidemiological tool for contact tracing. $\mathrm{R}$ package version 0.6.9. http://CRAN.Rproject.org/package=EpiContactTrace.

\section{Population-attributable fractions}

Population-attributable fractions (AF) were calculated for each variable using Levin's formula (Levin, 1953). Equation 2 shows an application with the ID variable.

$$
A F_{I D}=\frac{P_{I D} \times\left(O R_{I D}-1\right)}{1+P_{I D} \times\left(O R_{I D}-1\right)}
$$

(equation 2)

where $A F_{I D}$ is the AF of the ID variable, $P_{I D}$ the proportions of herds that receives animals from at least one herd and $O R_{I D}$ the $\mathrm{OR}$ assessing the relation between animal movements and the risk for a herd to become positive.

\section{Results}

\section{Spatial distribution of $Q$ fever infected dairy herds}

The prevalence of herds with antibodies against C. burnetii in the BTM in Finistère and on the Gotland Island was $69.4 \%$ and $55.2 \%$, respectively. One cluster of positive dairy herds was identified in the northwestern part of Finistère (relative risk $=1.2$ ). None was identified on Gotland (Fig. 1).

\section{Risk factor analysis}

\section{Selection of periods for ID calculation}

The risk for a herd to be detected as positive decreased when accounting for animal movements long before the date of testing (Figs. $2 \mathrm{a}$ and $2 \mathrm{c}$ ). Both in France and Sweden, the goodness of fit highly increased (AIC decreased) when considering animal movements during a 2 -year period compared to only 1 year before the ELISA test (Figures $2 \mathrm{~b}$ and $2 \mathrm{~d}$ ). While in France the goodness of fit stabilised afterwards, it increased in Sweden until reaching a stable value. Both evolutions indicate that accounting for more than 2 years before testing does not result in an increase of the goodness of fit. Based on these results, IDs for both areas were calculated during a 2-year period before the test was carried out, i.e. from January 2010 to December 2011 for the French dairy herds and from January 2009 to December 2010 for the Swedish ones.

\section{Descriptive statistics}

The local cattle density varied markedly between the two studied areas. The local cattle density was higher in Finistère District than on the Gotland 
(a)

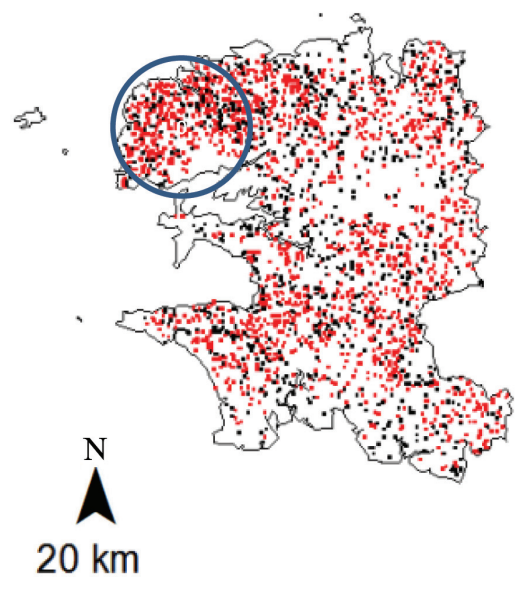

(b)

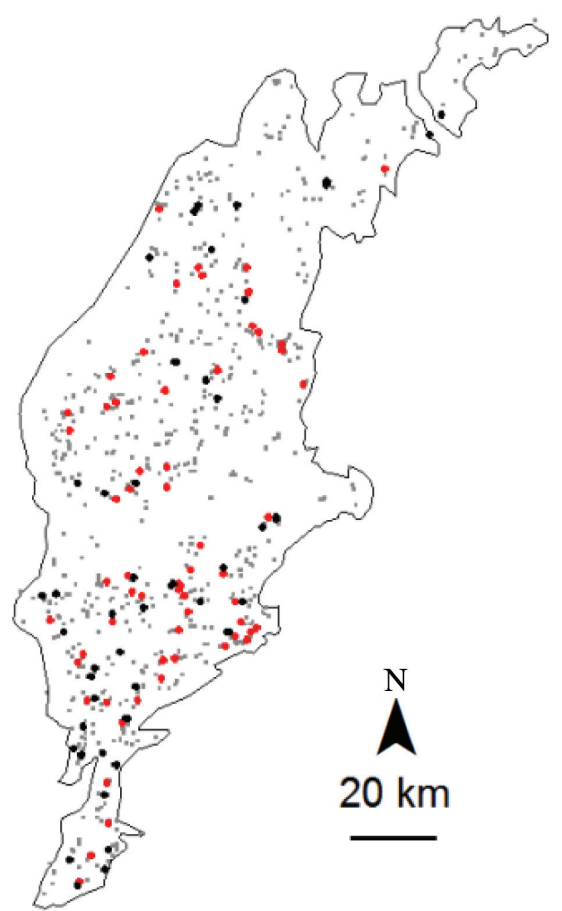

Fig. 1. Location of all the dairy herds in (a) the Finistère District, France $(\mathrm{n}=2,829)$ and (b) on the Gotland Island, Sweden $(\mathrm{n}=246)$. The red and black dots represent herds that were positive and negative, respectively, when tested for antibodies to C. burnetii in bulk tank milk (BTM). The BTM was collected in May 2012 in France and in June 2011 on Gotland. The grey dots (in 1b) represent dairy herds that were not included in the study. The blue circle corresponds to a location with higher risk for positivity $(\mathrm{RR}=1.21)$.

Island (Fig. 3a); the average local cattle densities were 75.4 and 24.9 animals per $\mathrm{km}^{2}$, respectively. During the 2-year period before the ELISA tests, a herd received animals directly from a maximum of 25 other herds (Fig. 3b), with a similar distribution of the ID parameter in the Finistère and on Gotland (Finistère: median $=0$, mean $=1.1$; Gotland Island: median $=1$, mean $=2.2$ ).

(a)
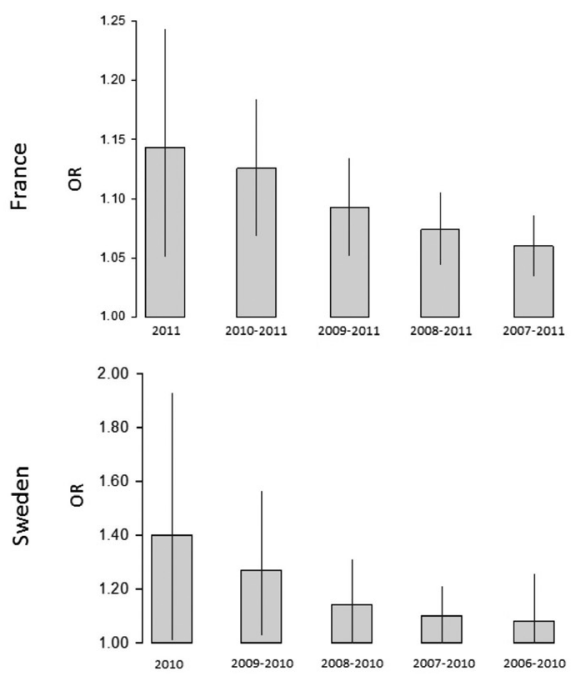

\section{Multivariable analysis}

The risk of detecting antibodies against C. burnetii in the BTM was associated with both animal movements and the local cattle density (Table 1 ). The higher the number of other herds from which a herd received animals, the higher the risk to be detected as positive. A first model was run (results not shown) to define the (b)
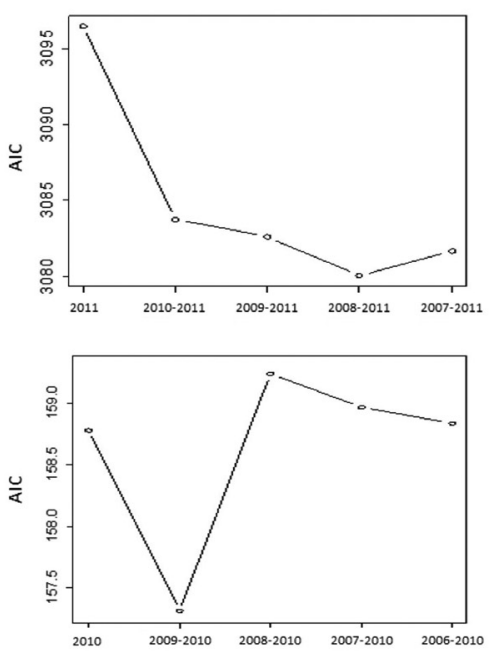

Fig. 2. Relationship between the risk for a herd to be found positive with respect to C. burnetii in bulk tank milk (BTM) and animal movements in France and Sweden assessed by: (a) odds ratio (OR) and (b) Akaike information criterion (AIC). 

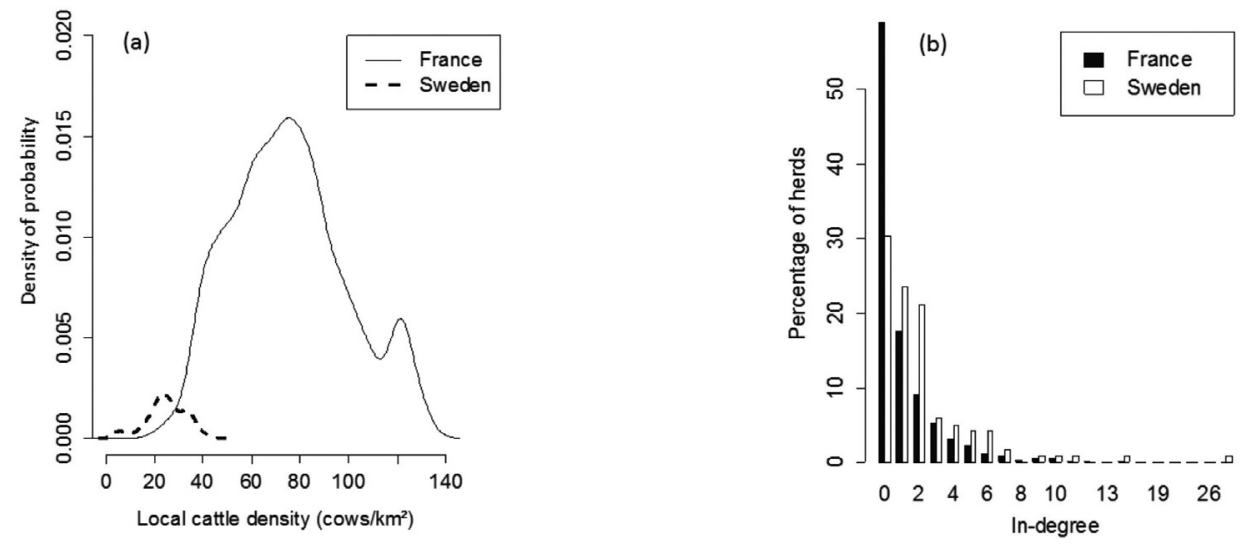

Fig. 3. Distribution by country of the local cattle density expressed as cows per $\mathrm{km}^{2}$ (a) and distribution of the in-degree parameter (ID) (b). The IDs (calculated during a 2-year time period before the ELISA tests) correspond to the number of herds from which each herd received animals directly.

reference population for the local cattle density. For the three categories 0-20, 20-40 and 40-60 cows per $\mathrm{km}^{2}$, there was no association with the risk for a herd to become positive. Consequently, all the herds located in areas with less than 60 cows per $\mathrm{km}^{2}$ were considered the reference population. The risk for a herd to be detected as positive increased almost linearly between 60 and 100 cows per $\mathrm{km}^{2}$ and then stabilised at an OR around 2.3. Finally, potential interactions between the explanatory variables were tested. None of them was associated with the risk for a herd to become positive.

\section{Population-attributable fractions}

Overall, attributable fractions of the animal movement and the local cattle density were $15.6 \%$ and $34.3 \%$, respectively (Table 1 ). The AF corresponding to the local cattle density in Sweden was null because the density was lower than 60 cows per $\mathrm{km}^{2}$ for all the herds (herds for which the local density was below this value were considered as the reference population). Concerning the ID variable, the AF was higher in Sweden than in France.

\section{Discussion}

This study confirms that the risk of C. burnetii infection is influenced both by animal movements and by the closeness between cattle herds. Overall, the strengths of the associations were similar for the two risk factors. Moreover, for both of them, the risk for a herd of becoming infected almost linearly increased with exposure levels. Only one cluster of infected herds was detected in the north-western part of Finistère.

Table 1. Distribution of herds regarding exposure levels to C. burnetii. A total of 119 dairy herds were tested in Sweden (June 2011) and 2,829 dairy herds were tested in in France (May 2012).

\begin{tabular}{|c|c|c|c|c|c|c|}
\hline Variable & $\begin{array}{c}\text { Exposure } \\
\text { level }\end{array}$ & $\begin{array}{c}\text { Population } \\
(\%)\end{array}$ & $\begin{array}{c}\text { OR } \\
(95 \% \mathrm{CI})^{*}\end{array}$ & P-value & $\begin{array}{c}\mathrm{AF}^{* *} \text { per } \\
\text { variable }\end{array}$ & $\begin{array}{l}\mathrm{AF}^{* *} \text { per } \\
\text { country }\end{array}$ \\
\hline \multirow[t]{3}{*}{ In-degree (ID) } & 0 & 57.7 & 1 & & & France: 15.1 \\
\hline & $1-3$ & 32.4 & $1.27(1.05-1.53)$ & 0.01 & 15.6 & Sweden: 25.1 \\
\hline & $>4$ & 9.3 & $2.31(1.65-3.24)$ & $<0.001$ & & \\
\hline Local cattle & $0-60$ & 32.1 & 1 & & & France: 35.2 \\
\hline \multirow[t]{4}{*}{ density (DENS) } & $60-80$ & 30.1 & $1.57(1.26-1.95)$ & $<0.001$ & & Sweden: 0 \\
\hline & $80-100$ & 22.3 & $1.77(1.39-2.25)$ & $<0.001$ & 34.3 & \\
\hline & $100-120$ & 8.7 & $2.26(1.60-3.19)$ & $<0.001$ & & \\
\hline & $120-140$ & 6.8 & $2.34(1.59-3.43)$ & $<0.001$ & & \\
\hline \multirow[t]{2}{*}{ Country } & France & 96 & 1 & & & \\
\hline & Sweden & 4.0 & $0.67(0.44-1.004)$ & 0.052 & & \\
\hline
\end{tabular}

*Odds ratio with $95 \%$ confidence interval; **Population attributable fraction (calculated using OR assessing the risk associated with binary exposures for the same reference populations and corresponding population proportions). 
A higher risk of infection for herds located in areas characterised by high cattle density compared to areas with low density highlights the significance of local propagation of the bacterium between herds. It was assumed that the underlying transmission process was mainly influenced by wind which could contribute to the contamination via inhalation of still susceptible animals. Another transmission route involving ticks exists (Sting et al., 2004; Castillo et al., 2010), but it has been considered not essential in the cycle of C. burnetii infection in livestock (Babudieri, 1959; European Food Safety Authority, 2010) and was thus considered as negligible compared to the risk factors studied here. A similar association with cattle density was found for dairy goat farms. A higher risk of serological Q fever infection was found in farms located in municipalities with cattle density $>100$ cattle per $\mathrm{km}^{2}$ compared to farms in municipalities with less than 100 cattle per $\mathrm{km}^{2}$ (Schimmer et al., 2011).

The local density was here calculated considering all the cattle, both beef and dairy, within $5 \mathrm{~km}$ around each herd. This choice was based on a study investigating the epidemiological link between a cluster of human Q fever cases and Q fever-positive dairy goat farms, which suggested an effective range of air-borne C. burnetii spread of $<5 \mathrm{~km}$ (Schimmer et al., 2010). In that study, people living within $5 \mathrm{~km}$ from a positive farm had a higher risk for $\mathrm{Q}$ fever than those living further away.

Animal movements within $5 \mathrm{~km}$ of the herd of origin may confound the effect of local transmission. Thirty and $23 \%$ of the movements in Finistère and on the Island of Gotland occurred within $5 \mathrm{~km}$, respectively. To test this possible confusion, the ID variable was split into two variables depending on the distance between the herd of origin and the receiving herd: transfer within or $5 \mathrm{~km}$ of the herd of origin. In terms of magnitude, one additional transfer had the same effect on the risk for a herd to become positive, regardless of the distance between the herd of origin and the receiving herd (results not shown). Moreover, adding the local density in the model did not change the effect of animal movements. These results indicated that local animal movements and local cattle density play distinct roles in the transmission of the bacterium between herds.

The population-attributable fraction assesses the proportion of cases attributable to a certain risk factor. In other words, it corresponds to the fraction of the cases in the population that would not have occurred, had the risk factor in question been absent. In this study, the overall proportion of cases attributa- ble to closeness of infected neighbourhoods was twice that the proportion attributable to animal transfers. It reflects the fact that overall, around $70 \%$ of them were located in areas with a medium to high cattle density, while more than half of the herds did not have contact with any other herd during the 2-year period before being tested. On Gotland, the local cattle density was not high enough to influence this risk, so only animal movements could be investigated. The fact that it was shown to contribute to the number of positive samples here is consistent with the fact that no cluster was detected in the Swedish site.

A limitation of the study concerns the risk of misclassification bias by relying on the detection of antibodies against C. burnetii and not the pathogen itself. Thus, some of the herds testing positive might not be infective on the date when the BTM test was carried out but much earlier, which could have resulted in the inclusion in the ID parameter of some movements that actually occurred after the infective stage. In that case, exposure (animal movement) and infection may not have coincided. In this context, we made the decision to account only for direct animal movements, and not for indirect ones that could have been taken into account using another network parameter, the ingoing infection chain (Wasserman et al., 1994). To circumvent this limitation, an interesting perspective would be to measure the infection status by the polymerase chain reaction (PCR), which allows detecting bacterial DNA (Frazier et al., 1990). Then, it would be possible to investigate the risk for a herd to be newly detected as infected taking into account animal movements occurring within the months preceding this changing status. Moreover, it would also be interesting to consider the risk level of the herd of origin (high risk, low risk herd or unknown status). Furthermore, the closeness between cattle herds was used here to indirectly investigate the role of wind. Another extension of this work would be to investigate the association between $\mathrm{Q}$ fever incidence and meteorological data such as wind direction and speed and other weather-related conditions.

While animal movements can be controlled to limit the infection spread, cattle density cannot be modified in the same way. Therefore, the control measure to be implemented could vary according to the local cattle density. For example, for herds located in areas with high cattle density, vaccination could be an appropriate control measure. Indeed, vaccination of dairy cows is associated with a reduction in C. burnetii bacterial load measured both at the individual and herd levels that could reduce the transmission of the bacterium (Taurel et al., 2012). In all areas, prevention should 
also be based on herd diagnosis for herds from which animals are sold (for instance, requirement of negative test results, both by PCR and ELISA on BTM).

\section{Conclusion}

The neighbourhood between cattle herds and animal movements both influence the risk for a herd to be infected. The great influence of neighbourhood in high density areas suggests that wind-borne spread plays a major role. The control measures to be implemented in herds at the regional level could differ according to the cattle density (not preventable) and the animal movements (preventable through herd testing).

\section{Acknowledgements}

This work was supported by the French Research Agency program Investments for the future, project ANR-10-BINF-07 (MIHMES). The authors thank Jenny Frössling, Raphaël Guatteo and Jean-Yves Audiart for their contributions. The Swedish data collection was supported by the C. F Lundström Foundation.

\section{References}

Arricau-Bouvery N, Rodolakis A, 2005. Is Q fever an emerging or re-emerging zoonosis? Vet Res 36, 327-349.

Babudieri B, 1959. Q fever: a zoonosis. Adv Vet Sci 5, 81-182. Berri M, Laroucau K, Rodolakis A, 2000. The detection of Coxiella burnetii from ovine genital swabs, milk and fecal samples by the use of a single touchdown polymerase chain reaction. Vet Microbiol 72, 285-293.

Castillo L, Fernandez-Llario P, Carranza Almansa J, Bermejo F, Hermoso de Mendoza J, 2010. First seropositive cases of Coxiella burnetii in red deer populations in the southwest Iberian peninsula. J Zoo Wildl Med 41, 468-473.

Christley RM, Pinchbeck GL, Bowers RG, Clancy D, French NP, Bennett R, Turner J, 2005. Infection in social network: using network analysis to identify high-risk individuals. Am J Epidemiol 162, 1024-1031.

European Food Safety Authority, 2010. Panel on animal health and welfare (AHAW). EFSA Journal 8, 1595. Available at: http://www.efsa.europa.eu/it/efsajournal/doc/1595.pdf (accessed on March 2013).

Frazier ME, Mallavia LP, Samuel JE, Baca OG, 1990. DNA probes for the identification of Coxiella burnetti strains. Ann N Y Acad Sci 590, 445-458.

Frössling J, Ohlson A, Björkman C, Hakansson N, Nöremark M, 2012. Application of network analysis parameters in riskbased surveillance - examples based on cattle trade data and bovine infections in Sweden. Prev Vet Med 105, 202-208.
Guatteo R, Beaudeau F, Berri M, Rodolakis A, Joly A, Seegers $\mathrm{H}, 2006$. Shedding routes of Coxiella burnetii in dairy cows: implications for detection and control. Vet Res 37, 827-833.

Guatteo R, Beaudeau F, Joly A, Seegers H, 2007. Coxiella burnetii shedding by dairy cows. Vet Res 38, 849-860.

Kulldorff M, 1997. A spatial scan statistic. Commun Stat Theory Methods 26, 1481-1496.

Kulldorff M, Information Management Services, 2009. Inc. SaTScanTM v8.0: software for the spatial and space-time scan statistics. Available at: http://www.satscan.org (accessed on March 2013).

Levin ML, 1953. The occurrence of lung cancer in man. Acta Unio Int Contra Cancrum 9, 531-541.

Nöremark M, Hakansson N, Sternberg Lewerin S, Lindberg A, Jonsson A, 2011. Network analysis of cattle and pig movements in Sweden: measures relevant for disease control and risk based surveillance. Prev Vet Med 99, 78-90.

R Development Core Team, 2012. R: a language and environment for statistical computing. R Foundation for Statistical Computing. Vienna: R Foundation for Statistical Computing. Schimmer B, Luttikholt S, Hautvast JL, Graat EA, Vellema P, Duynhoven YT, 2011. Seroprevalence and risk factors of Q fever in goats on commercial dairy goat farms in the Netherlands, 2009-2010. BMC Vet Res 7, 81.

Schimmer B, Ter Schegget R, Wegdam M, Zuchner L, de Bruin A, Schneeberger PM, Veenstra T, Vellema T, van der Hoek W, 2010. The use of a geographic information system to identify a dairy goat farm as the most likely source of an urban Q-fever outbreak. BMC Infect Dis 10, 69.

Sting R, Breitling N, Oehme R, Kimmig P, 2004. The occurrence of Coxiella burnetii in sheep and ticks of the genus Dermacentor in Baden-Wuerttemberg. Dtsch Tierarztl Wochenschr 111, 390-394.

Taurel AF, Guatteo R, Beaudeau F, 2012. PhD thesis, "Assessment of the effectiveness of medical measures to control Coxiella burnetii infection in dairy cattle herds". Université de Nantes, Angers, Le Mans, 175 pp.

Tissot-Dupont H, Amadei MA, Nezri M, Raoult D, 2004. Wind in November, Q fever in December. Emerg Infect Dis 10, 12641269.

Tissot-Dupont H, Torres S, Nezri M, Raoult D, 1999. Hyperendemic focus of Q fever related to sheep and wind. Am J Epidemiol 150, 67-74.

Wasserman S, Faust K, Iacobucci D, Granovetter M, 1994. Social network analysis: methods and applications (structural analysis in the social sciences). Cambridge, Cambridge University Press, 819 pp.

Welsh HH, Lennette EH, Abinanti FR, Winn JF, Kaplan W, 1959.Q fever studies. XXI. The recovery of Coxiella burnetii from the soil and surface water of premises harboring infected sheep. Am J Hyg 70, 14-20. 